\title{
Bimodal spectroscopic evaluation of ultra violet-irradiated mouse skin inflammatory and pre-cancerous stages: instrumentation, spectral feature extraction/selection and classification (k-NN, LDA and SVM)
}

\author{
G. Díaz-Ayil ${ }^{1}$, M. Amouroux ${ }^{1}$, W.C.P.M. Blondel ${ }^{1}$, G. Bourg-Heckly², A. Leroux ${ }^{3}$, F. Guillemin ${ }^{1,3}$ and Y. Granjon $^{1}$ \\ 1 Centre de Recherche en Automatique de Nancy, Nancy-University, CRAN UMR 7039 CNRS-Nancy University, 2 avenue de la \\ Forêt de Haye, 54516 Vandoeuvre-les-Nancy, France \\ ${ }^{2}$ Laboratoire de Biophysique Moléculaire, Cellulaire et Tissulaire, BioMoCeTi UMR 7033 CNRS-Université Pierre et Marie Curie \\ Paris 6, Genopole Campus 1, 5 rue Henri Desbruères, 91030 Evry cedex, France \\ ${ }^{3}$ Centre Alexis Vautrin, 6 avenue de Bourgogne, 54511 Vandoeuvre-lès-Nancy cedex, France
}

Received: date / Revised version: date

\begin{abstract}
This paper deals with the development and application of in vivo spatially-resolved bimodal spectroscopy (AutoFluorescence AF and Diffuse Reflectance DR), to discriminate various stages of skin precancer in a pre-clinical model (UV-irradiated mouse): Compensatory Hyperplasia $\mathrm{CH}$, Atypical Hyperplasia $\mathrm{AH}$ and Dysplasia D. A programmable instrumentation was developed for acquiring AF emission spectra using 7 excitation wavelengths: 360, 368, 390, 400, 410, 420 and $430 \mathrm{~nm}$, and DR spectra in the $390-720 \mathrm{~nm}$ wavelength range. After various steps of intensity spectra preprocessing (filtering, spectral correction and intensity normalization), several sets of spectral characteristics were extracted and selected based on their discrimination power statistically tested for every pair-wise comparison of histological classes. Data reduction with Principal Components Analysis (PCA) was performed and 3 classification methods were implemented (k-NN, LDA and SVM), in order to compare diagnostic performance of each method. Diagnostic performance was studied and assessed in terms of Sensibility (Se) and Specificity ( $S p)$ as a function of the selected features, of the combinations of 3 different inter-fibres distances and of the numbers of principal components, such that: $S e$ and $S p \approx 100 \%$ when discriminating $\mathrm{CH}$ vs. others; $S p \approx 100 \%$ and $S e$ $>95 \%$ when discriminating Healthy $v s$. AH or D; $S p \approx 74 \%$ and $S e \approx 63 \%$ for $\mathrm{AH} v s$. D.
\end{abstract}

Keywords: spectral data classification, in vivo spectroscopy, autofluorescence, diffuse reflectance.

PACS. 87.64.-t Spectroscopic and microscopic techniques in biophysics and medical physics - 87.80.-y Biophysical techniques (research methods) - 07.60.-j Optical instruments and equipment

\section{Introduction}

Because outdoor activities and esthetic considerations (such as skin tanning) are of growing interest in western countries, fair-skinned populations have been more and more exposed to sun light in the past decades. Since solar ultra-violet (UV) radiation is the main environmental cause of skin cancer, the incidence of the three major forms of skin cancer (melanoma, squamous cell carcinoma (SCC) and basal cell carcinoma) is growing. Even though most deaths are attributable to melanoma, SCC is the most common life-threatening cancer type [1]. In dermatology, diagnosis is based on direct visualisation of the lesion. Because such observation depends on personal experience of the physician, lesions may be falsely considered benign, may not undergo biopsy, and therefore may not be subjected to the reference diagnostic gold standard i.e. histo-pathology [2]. Currently, full diagnosis of suspicious skin lesions implies excision-biopsy of the lesion followed by anatomo-pathological examination. Such a diagnosis process is invasive and thus implies pain, increased morbidity and substantial damage to nearby healthy skin (esthetic inconvenience). Moreover the sensitivity of this method is limited because only a few tissue samples can be biopsied in a suspicious area. Noninvasive methods such as MRI, ultrasounds and spectroscopy have the potential to eliminate these problems and also to permit the monitoring of treatment effects [3]. Optical methods (imaging and spectroscopy) in the UV Visible - Near-Infra-Red spectral range are of particular interest because they are harmless (using the non-ionizing part of the electromagnetic spectrum) and sensitive to morphologic and functional modifications of biological 
tissues occuring during early stages of cancer development [4]. Furthermore to improve inter-observer agreement and diagnosis'accuracy (sensitivity and specificity), optical spectroscopy can be useful by providing physicians with more objective criteria. Spectroscopy methods are based on interactions of light with biological tissues. During the past twenty years, Diffuse Reflectance (DR) and AutoFluorescence (AF) spectroscopies have been the most investigated spectroscopy modalities [4]. DR spectroscopy is based on elastic scattering of photons within biological tissues. Elastic scattering is caused by variations in the refractive index of small particles in the medium, resulting in dispersion of light in all directions, without loss of energy, i.e. no wavelength shift between incident and reflected light. Different types and sizes of scattering centers can be found within the tissue: collagen fibers, cellular membranes and subcellular structures (nuclei, mitochondria) [5]. AF spectroscopy is based on the analysis of fluorescence emission by endogenous molecules, i.e. materials that are natural constituents of biological tissues (intra or extra-cellular metabolic products). Such endogenous fluorophores are usually excited in the 300-450 spectral range. These molecules are related to the stucture arrangement of cells and tissues (such as collagen, elastin, etc.) and to the cellular metabolism (such as $\mathrm{NAD}(\mathrm{P}) \mathrm{H}$, flavins, etc.). As a result, different morphofunctional conditions of the skin related to pathologic alterations such as cancer development can be characterized using either AF, DR or both [5]. As shown in previous works [6-8], combining both modalities (DR and AF) provides a more accurate diagnosis (increased sensitivity and specificity). A typical optical spectroscopy set-up includes a light source, an optical fiber bundle (including excitation and collection fibers) put in gentle contact with the skin and a spectrometer. Optical fibers allow spectroscopy to be used not only on skin but also on less accessible anatomical sites such as hollow inner organs (bladder, oesophagus, cervix, etc.) Most optical fibre bundles are currently used with different Collection-to-Excitation Fibre Separations (CEFS) and with various geometrical configurations in order to optimize signal collection [9, $10]$ and to probe tissues at various depths. Systems for DR spectroscopy (380-950 $\mathrm{nm}$ or 300-800 $\mathrm{nm}$ ) associated to AF excitations between 300 and $500 \mathrm{~nm}$ have been built and used in several studies [11-13]. They were composed of an arc-lamp and a monochromator in order to get a large number of fluorescence excitation wavelengths and wide spectral range for diffuse reflectance. These systems used 7 to 46 -fibre bundles and highly sensitive multi-track acquisition devices (intensified CCD). The spectroscopic set of acquired data consists in AF and DR spectra whose shape and intensity vary as a function of tissue structure and CEFS. Such a set of spectra represents a large amount of data to be reduced and analyzed. Various methods of processing spectral data were previously described. They ususally include three steps: i) features extraction: although full spectra can be directly processed, most of the time, specific spectral features (such as areas, peak intensities, ratios, etc.) are extracted; ii) spectral data set reduction based on factorial analysis mostly using Principal Component Analysis (PCA); iii) classification based on different types of supervised and non-supervised algorithms, the latter allowing to calculate sensitivity (Se) and Specificity (Sp), both being diagnostic parameters of clinical interest, with reference to histological classes. The choice of the method will influence the obtained values of sensitivity and specificity.

The aim of the current work is to assess bimodal spectroscopy's accuracy in classifying UV- irradiated mouse skin samples into four histological precancerous classes. The objectives are threefold: i) develop a bimodal spectroscopy instrumentation for in vivo acquisition of $\mathrm{AF}$ and DR spectra from mouse skin. Since the measurement protocol lasted for several months, calibration and normalization procedures were developed to account for the system thoughput variations (light source intensity, acquisition set-up) over the 30 -week period of time; ii) extract an extended set of "a priori" spectral data, then select the most discriminative ones with reference to each pair-wise discrimination; iii) investigate whether improvements in classification performance could be obtained using different linear classification methods (namely k-Nearest Neighbor, Linear Discriminant Analysis or Support Vector Machine).

\section{Materials and Methods}

\subsection{Bimodal multi-excitation spectroscopy set-up}

Figure 1 presents a scheme of the developed multi-excitation-collection spatially resolved spectroscopic system. Source light injected in the excitation fibre (Fexc) comes from a short-arc 300W Xenon lamp with a parabolic mirror (Eurosep, France). The lamp emits mainly in the 300-800 nm spectral range. An anti-caloric filter (F1) protects optical components from heating by infra-red wavelengths. Light from the lamp is focused at the entrance of the optical excitation fiber (Fexc) using the convex lens L1. A bandpass filter $\mathrm{F} 2$ is used to limit the excitation spectral range to $350-450 \mathrm{~nm}$ in order to avoid at best the transmission of light interfering with the endogenous fluorescence emission, mainly emit in the $450-700 \mathrm{~nm}$ range. The set-up consists in a dynamically-tunable excitation fluorescence system: center wavelength and Full Width at Half Maximum (FWHM) can be set thanks to the combination of linearly variable high-pass (FL1) and low-pass (FL2) filters. Both filters are fixed on translation stages (UMR 8.51, Newport) controlled by a motorized micrometric positioning system (actuators LTA-HS, $0.1 \mu \mathrm{m}$ precision and XPS controller, Newport) used to tune excitation peaks (between 350 and $460 \mathrm{~nm}$ ) as well as illumination bandwidths (FWHM between 15 and $25 \mathrm{~nm}$ ) in a precise and repeatable way. For diffuse reflectance, three illumination spectral bands were selected: 365-545, 450-640 and $550-740 \mathrm{~nm}$. We used a 37-optical fibre bundle (available CEFS are between 250 and $1500 \mu \mathrm{m}$ ). Up to 13 spectra can be acquired simultaneously thanks to a multi-track imaging spectrograph equipped with a UV back-illuminated 


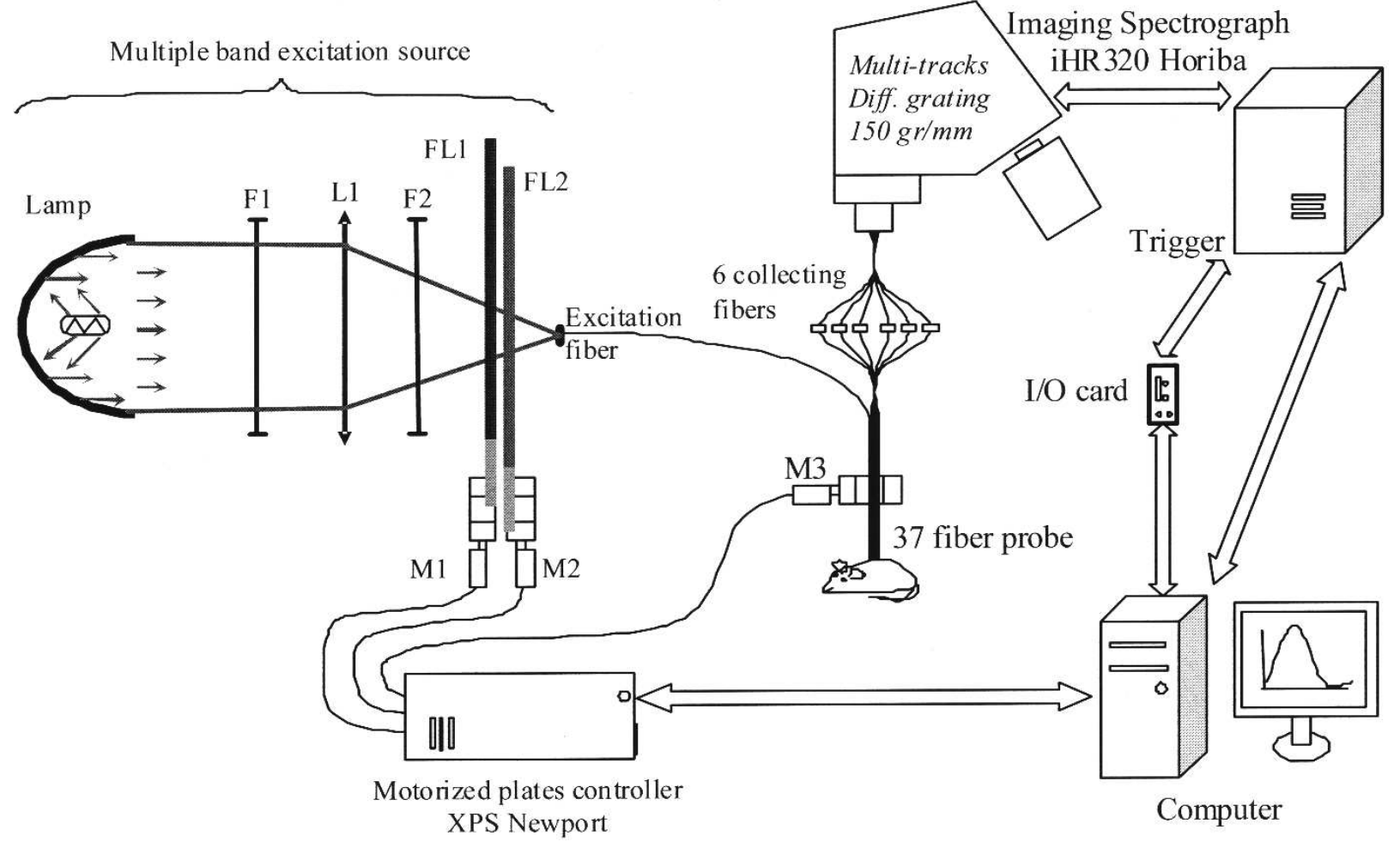

Fig. 1. Scheme of the bimodal spectroscopy device developed. Short arc lamp (300W Xenon), F1: anticaloric filter, L1: convex lens, F2: bandpass filter, FL1 and FL2: high-pass and low-pass linearly variable filters, Fexc: exciting optical fibre, M1, M2 and M3: motorized micrometric positioning actuators used for linear displacement.

CCD (iHR 320, Horiba Jobin Yvon, France) and an emission filter-wheel with various filters used to reject the backscattered excitation light. For our application, we selected 3 different CEFS: 271,536 and $834 \mu \mathrm{m}$ so as to probe the tissue at various depths; as mentionned by Katika et al. [14] for instance, a $250 \mu \mathrm{m}$-CEFS provides information from a $100 \mu \mathrm{m}$-depth in the skin at $442 \mathrm{~nm}$. Integration times were $500 \mathrm{~ms}$ and $50 \mathrm{~ms}$ for AF and DR respectively. Spectra were all acquired using a diffraction grating (150 lines $/ \mathrm{mm}$ ) blazed at $600 \mathrm{~nm}$.

A dedicated software was developed using Visual $\mathrm{C}++$ 6.0 (Microsoft corporation) in order to control the translation stage displacements (for excitation peaks and bands tuning) and to synchronize the excitation system with the iHR320 spectrometer acquisition using external triggering.

The system automatically switches from each acquisition wavelength to the following one. Such a combination of procedures can be repeated as many times as required. For each excitation, 3 spectra were acquired and averaged in order to improve signal-to-noise ratio.

\subsection{Skin cancer animal model and experimental protocol}

The device being described in the current paper was developed in order to diagnose pre-cancerous stages of Squamous Cell Carcinomas (SCC) which is the most letal form of non melanoma skin cancers. Skin tumours are induced by UV-irradiation of SKH1 mice (albino and hairless). We chose this way of inducing tumours (also called 'spontaneous' tumours) in order to remain as close to human etiology as possible. Twenty-eight mice were divided into three groups: group I included 8 sham-irradiated mice (control group), group II included 10 mice irradiated for 1 month (50 $\mathrm{mJ} / \mathrm{cm}^{2}$ weekly dose) and group III included 10 mice irradiated for 6 months $\left(50 \mathrm{~mJ} / \mathrm{cm}^{2}\right.$ once a week for the first 15 weeks then twice a week for the rest of the irradiation protocol). Mice were anesthesized with a mix of Ketamine and Xylazine during spectroscopic measurements. During the 30-week protocol, 2 mice a week were regularly euthanized and back skin was sampled. Each piece of skin was further fixed in $10 \%$ formaldehyde solution and $5 \mu \mathrm{m}$-slices were stained with standard Hematoxylin and Eosin (to evaluate epidermis features) and specific Weigert staining (to evaluate dermal elastic fibers). Then, based on histo-pathological criteria, main precancerous mouse skin stages characteristics were identified and skin samples were further classified in each of the four following classes: Healthy $(\mathrm{H})$, Compensatoty Hyperplasia $(\mathrm{CH})$, Atypical Hyperplasia $(\mathrm{AH})$ and Dysplasia (D). Such a classification was made thanks to a pathologist evaluation of epidermis and dermis features: epidermis thickness, presence of mitosis in upper layers of epidermis, hyperkeratosis, nucleoted upper layers, heterogenous chromatin, cell layer disorganization, 

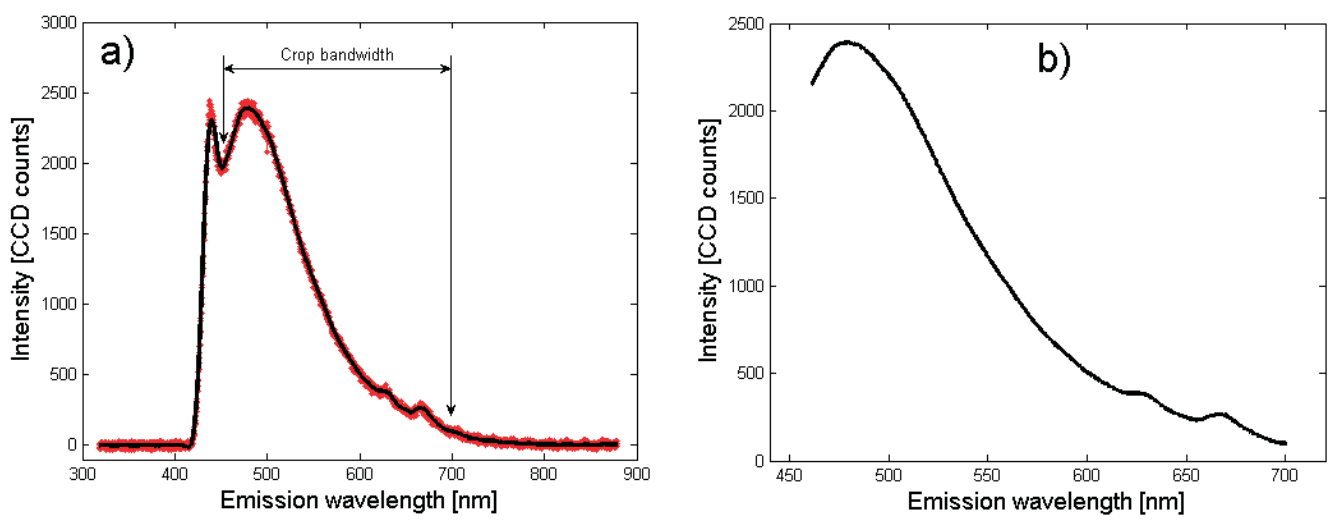

Fig. 2. Crop bandwidth, mean and filtered AF-410 nm emission. a) mean spectra (red), filtered spectra (black); b) crop bandwidth.

parakeratosis, dyskeratosis and elastosis.

Finally, the histological set of data used in the present contribution consisted of 84 healthy, 47 compensatory hyperplastic, 64 atypical hyperplastic and 57 dysplastic hyperplastic skin samples.

Colocalized AF and DR spectra were acquired on twelve spots of each mouse's back skin : six spots along the backbone on each side (left and right). Seven excitation wavelengths (360, 368, 380, 390, 400, 410, 420 and $430 \mathrm{~nm}$ ) were used for AF spectroscopy and three illumination bands (365-545, 450-640 and 550-740 nm) were used for DR spectroscopy. Three Collection to Excitation Fibers Separations (CEFS) were used: 271, 536 and $834 \mu \mathrm{m}$.

\subsection{Spectral Data Processing}

\subsubsection{Preprocessing (filtering, spectral correction, normalization)}

After building 3D-matrices made of three (number of CEFS) 2D-matrices of 30 intensity spectra each (7 excitations for AF and 3 DR illumination bands, $n=3$ for each), a 6-step preprocessing was performed on raw AutoFluorescence $(\mathrm{AF})$ spectra:

1. High amplitude narrow peak artefacts suppression: a $4 \mathrm{~nm}$-width (15 points) sliding window was applied over the entire intensity spectra. Artefacts are detected based on a variance variation of more than $400 \%$ between successive windows. Then parasitic points are suppressed based on a 30 time-averaging with the previous data point.

2. The raw spectral intensity curves acquired are affected by a random measurement noise characterized by a Gaussian distribution. In order to improve the signal signal-to-noise ratio [15,7] 3 acquisitions in a raw were systematically performed then averaged $(n=3$, maximum number compatible with acquisition time); by this way noise amplitude was reduced by $40 \%$.

3. Residual high frequency was removed using a polynomial smooth filtering (Stavizky-Golay) [16]. The window size and order of the filter were determined for each type of spectra (AF or DR) with reference to their specific shape characteristics. A $22 \mathrm{~nm}$ width window (2nd order) was applied to all AF spectra and 15 to $30 \mathrm{~nm}$ width window (2nd order) were used for DR depending on the spectra width band. The windows widths and filter order of the implemented filters were defined in particular to smooth "correctly"the informative narrowest parts of the curves while preserving the main shape (for instance porphyrin emission peaks of width $35-40 \mathrm{~nm}$ visible in the $620-690 \mathrm{~nm}$ band, figure $2 a$ ).

4. The curve of a typical AF emission spectrum (figure $2 \mathrm{a}$ ) acquired can be subdivised in 3 parts, the central part being the useful informative one. The lower wavelengths part may exhibit residual intensities due to the backscattered excitation light (although filtered), and no more AF signals is observed above 700 $\mathrm{nm}$. In order to only keep the useful part of the emission spectra acquired (figure $2 b$, crop bandwidth for $410 \mathrm{AF}$ ), we defined the following crop bandwidths of interest: 410-700nm for AF360 and AF368; 460-700nm for AF390, AF400 and AF410; 463-700nm for AF420; 476-700nm for AF430; and 390-720nm for DR.

5. The various components of the acquisition system i.e. optical probe, fibers, lenses, mirrors, diffraction gratings, CCD, have specific spectral responses which affect the shape and amplitude of the acquired spectra. Therefore a correction of the system throughput is needed and was applied thanks to a calibration lamp (HL 2000-CAL, Ocean Optics). Correction coefficients were calculated by dividing the experimentally measured calibration lamp spectrum (acquired with the same acquisition configurations as the ones that were used during mouse skin measurements) by the reference or calibrated spectrum of the lamp.

6. The excitation source (Lamp Xenon 300W) has a relative power output that decreases with the operation time. As measurement protocol lasted for several weeks, a correction method was implemented to take this diminution into account. Source light intensity variations were taken into account thanks to power measurements at the probe tip every day at start of 
the experiment (Powermeter 841PE and detector UV 818-UV, Newport Corp.). Corrections were performed thanks to the division of raw spectra by the excitation light power measured on the same day of experiment.

For Diffuse Reflectance (DR) spectra, preprocessing approach was the same as the one used for AF spectra up to step \# 4. In this case, once bandwidths of interest were defined, skin spectra were normalized by standard spectra acquired on a Lambertian surface (WS-1 Diffuse Reflection Standard, Ocean Optics) obtained at the shortest CEFS $(271 \mu \mathrm{m})$.

\subsubsection{Spectral features selection and data reduction}

Based on our own observations of the main intensity and shape variations of acquired spectra and on previously published studies [17-23], we extracted 762 spectral features from each of the 24 spectra acquired on each skin spot (7 AF + 1 DR at each of the 3 CEFS):

- Areas: under the spectrum between $\lambda_{a}$ and $\lambda_{b}\left(A_{\lambda a-\lambda b}\right)$, under the the spectrum between $\lambda_{a}$ and $\lambda_{b}+$ spectral intensity contribution $\left(A T_{\lambda a-\lambda b}\right)$, above the spectrum $\left(A A_{\lambda a-\lambda b}\right)$, and normalized areas $\left(A N_{\lambda a-\lambda b}\right)$

- Ratio of intensities at $\lambda_{a}$ and $\lambda_{b}\left(R_{\lambda a-\lambda b}\right)$ and ratio of areas $\left(A_{\lambda a-\lambda b} / A_{\lambda b-\lambda c}\right)$

- Spectral fitting residual $(F)$ : a 10th - degre polynomial curve was fitted on the entire spectra and residual value registered

- Slope between $\lambda_{a}$ and $\lambda_{b}\left(S_{\lambda a-\lambda b}\right)$ and normalized slope $\left(N S_{\lambda a-\lambda b}\right)$

- Median $(M e)$, mean $(M)$ and standard deviation $(S D)$ of the entire spectra

All these spectral characteristics for AF and DR are given in detail in Table 1.

A normality Lilliefors test was implemented in order to keep (for next statistical test) only the spectral characteristics with normal statistical distribution. Then, a TStudent test was performed between all pairs of histological classes to set 3 groups of spectral features that give a pair-wise discrimination of either 6,5 or 4 pairs of histological skin classes (Table 2). Spectral features were extracted for each of the 3 CEFS: 271 (D1), 536 (D2) and 834 (D3) $\mu \mathrm{m}$.

In order to test spatially-resolved bimodal spectroscopy's accuracy in discriminating either 6,5 or 4 pairs of histological classes, we combined spectral features as indicated in Table 2: (D1 and D2), (D1 and D3), (D2 and D3) and (D1 and D2 and D3).

Once all the spectral features combinations were established, data were mean centered and Principal Component Analysis (PCA) was performed in order to reduce data set to a lower dimension and to provide uncorrelated data (Principal Components: PCs) for classification.

\subsubsection{Classification}

The results of three types of linear classification algorithm (k-NN, LDA and SVM) were compared. A super-
Table 2. Number of characteristics finally selected after statistical tests using each of the CEFS combinations of spatiallyresolved bimodal spectroscopy (each distance D1, D2, D3 and their 4 possible combinations) for spectral features that allowed a pair-wise discrimination of either 6,5 or 4 pairs of histological skin classes

\begin{tabular}{l|ccccccc}
\hline Distance & D1 & D2 & D3 & D1+D2+D3 & D1+D2 & D1+D3 & D2+D3 \\
\hline 6 pairs & 6 & 2 & 5 & 13 & 8 & 11 & 7 \\
S 5 pairs & 44 & 59 & 83 & 186 & 103 & 127 & 142 \\
- 4 pairs & 83 & 98 & 125 & 306 & 181 & 208 & 223 \\
\hline
\end{tabular}

vised classification was performed based on the results of histological analysis. The performance of this classification was expressed in the form of Sensibility Se (number of well classified pathologic samples) and Specificity $S p$ (number of well classified healthy samples) calculation.

k-Nearest Neighbors (k-NN) is a simple classification method giving good results with normal and non normal populations [24]. Using the Euclidean distance measure, the distances between validation data set and the calibration data set is computed with $\mathrm{k}$ neighbors samples number, then each measurement in the validation set is assigned the majority class belonging to its closest $\mathrm{k}$ neighbors in the training set. A difficulty for this method is the determination of the appropriate k-value, this is the reason why we proposed to test a wide range of values (typically 1 to 15).

The Linear Discriminant Analysis (LDA) aims at defining a set of axes (so-called linear discriminant functions) that best represent the distances existing between groups of data with reference to their variances. This method maximizes the ratio of between-class variance to the withinclass variance in any particular data set thereby guaranteeing maximal separability. It requires the data set to be normally distributed [25].

The Support Vector Machines (SVM) applies a transformation to the data (linear or nonlinear) in a greater dimension space, then applies a linear discrimination to separate the data. The objective of SVM is to obtain an optimal separating hyper-plane that maximizes the margin between two classes in a multidimensional data space. SVM was designed to be particularly effective in developing a reliable classifier from a training set with a small sample size [26-28].

For each of the three classification methods, algorithms to discriminate the 6 pairs of histological classes $(\mathrm{H}$ vs. $\mathrm{CH}$, $\mathrm{H} v$ s. AH, Hvs. D, CHvs. AH, CH vs. D and AH vs. D) were developed. True positives (pathologic skin samples that were correctly classified) and true negatives ("healthy" skin samples that were correctly classified) were used to evaluate sensitivity and specificity of the classification method. In order to test the robustness (generalization capacity), each algorithm was applied on 100 training and validation cycles with random selection of $66 \%$ of spectra as training set of data and the $34 \%$ left kept as the validation set of data.

$\mathrm{k}-\mathrm{NN}$ algorithms were performed using several combinations of number of neighbors (k) and Principal Com- 
Table 1. Spectral characteristics extracted for spectra acquired for each Autofluorescence excitation (e.g. AF-360 for the $360 \mathrm{~nm}$ excitation) and for DR spectra. Total area (AT), Normalized Area (AN) Normalized Slope (NS), Slope (S), Ratio (R) Mean (M), Standard Deviation (SD), Median (Me), Fitting Residual (F)

\begin{tabular}{|c|c|}
\hline$\lambda_{\text {exc }(n m)}$ & Spectral characteristics \\
\hline $\begin{array}{l}\text { AF-360 and } \\
A F-368\end{array}$ & $A T_{410-700}, N S_{410-445}, N S_{410-700}, S_{410-445}, S_{410-700}, R_{455-595}, A_{410-500} / A T_{500-700}, M, S D, M e$ and $F$ \\
\hline AF-390 & $\begin{array}{l}A T_{460-700}, A T_{460-545}, A_{460-545}, A N_{460-545}, A T_{615-650}, A_{615-650}, A N_{615-650}, A T_{655-690}, A_{655-690}, A N_{655-690} \\
N S_{460-700}, N S_{460-545}, N S_{615-650}, N S_{655-690}, N S_{460-470}, N S_{470-595}, N S_{633-670}, N S_{470-633}, S_{460-700}, S_{460-545} \\
S_{615-650}, S_{655-690}, S_{460-470}, S_{470-595}, S_{633-670}, S_{470-633}, R_{470-595}, R_{470-633}, R_{545-633}, R_{470-670}, R_{450-670} \\
R_{633-670}, A_{615-650} / A_{655-690}, A_{460-545} / A_{545-700}, M, S D, M e \text { and } F\end{array}$ \\
\hline AF-400 & $\begin{array}{l}A T_{460-700}, A T_{460-550}, A_{460-550}, A N_{460-550}, A T_{615-650}, A_{615-650}, A N_{615-650}, A T_{655-690}, A_{655-690}, A N_{655-690} \\
N S_{460-700}, N S_{460-550}, N S_{615-650}, N S_{655-690}, N S_{460-475}, N S_{485-595}, N S_{633.5-669}, N S_{485-633.5}, S_{460-700}, S_{460-550} \\
S_{615-650}, S_{655-690}, S_{460-475}, S_{485-595}, S_{633.5-669}, S_{485-633.5}, R_{485-595}, R_{485-633.5}, R_{550-633.5}, R_{485-669}, R_{550-669} \\
R_{633.5-669}, A_{615-650} / A_{655-690}, A_{460-550} / A_{550-700}, M, S D, M e \text { and } F\end{array}$ \\
\hline AF-410 & $\begin{array}{l}A T_{460-700}, A T_{460-550}, A_{460-550}, A N_{460-550}, A T_{615-650}, A_{615-650}, A N_{615-650}, A T_{655-690}, A_{655-690}, A N_{655-690} \\
N S_{460-700}, N S_{460-550}, N S_{615-650}, N S_{655-690}, N S_{460-485}, N S_{492-595}, N S_{633-668}, N S_{492-633}, S_{460-700}, S_{460-550} \\
S_{615-650}, S_{655-690}, S_{460-485}, S_{492-595}, S_{633-668}, S_{492-633}, R_{492-595}, R_{492-633}, R_{550-633}, R_{492-668}, R_{550-668} \\
R_{633-668}, A_{615-650} / A_{655-690}, A_{460-550} / A_{550-700}, M, S D, M e \text { and } F\end{array}$ \\
\hline AF-420 & $\begin{array}{l}A T_{463-700}, A T_{463-550}, A_{463-550}, A N_{463-550}, A T_{615-650}, A_{615-650}, A N_{615-650}, A T_{655-690}, A_{655-690}, A N_{655-690} \\
N S_{463-700}, N S_{463-550}, N S_{615-650}, N S_{655-690}, N S_{463-490}, N S_{505-595}, N S_{633-668}, N S_{505-633}, S_{463-700}, S_{463-550} \\
S_{615-650}, S_{655-690}, S_{463-490}, S_{505-595}, S_{633-668}, S_{505-633}, R_{505-595}, R_{505-633}, R_{550-633}, R_{505-668}, R_{550-668} \\
R_{633-668}, A_{615-650} / A_{655-690}, A_{463-550} / A_{550-700}, M, S D, M e \text { and } F\end{array}$ \\
\hline AF-430 & $\begin{array}{l}A T_{476-700}, A T_{476-560}, A_{476-560}, A N_{476-560}, A T_{615-650}, A_{615-650}, A N_{615-650}, A T_{655-690}, A_{655-690}, A N_{655-690} \\
N S_{476-700}, N S_{476-560}, N S_{615-650}, N S_{655-690}, N S_{476-490}, N S_{507-595}, N S_{633-669}, N S_{507-633}, S_{476-700}, S_{476-560} \\
S_{615-650}, S_{655-690}, S_{476-490}, S_{507-595}, S_{633-669}, S_{507-633}, R_{507-595}, R_{507-633}, R_{560-633}, R_{507-668}, R_{560-669} \\
R_{633-669}, A_{615-650} / A_{655-690}, A_{476-560} / A_{560-700}, M, S D, M e \text { and } F\end{array}$ \\
\hline DR 390-720 & $\begin{array}{l}A T_{390-710}, A T_{390-450}, A A_{390-450}, A T_{430-540}, A A_{500-600}, A T_{500-600}, N S_{390-720}, N S_{390-450}, N S_{500-600} \\
N S_{390-600}, N S_{430-540}, N S_{470-600}, N S_{580-600}, N S_{640-720}, N S_{430-450}, S_{390-720}, S_{390-450}, S_{500-600}, S_{390-600} \\
S_{430-540}, S_{470-600}, S_{580-600}, S_{640-720}, S_{430-450}, R_{390-720}, R_{390-450}, R_{500-600}, R_{390-600}, R_{430-540}, R_{470-600} \\
R_{600-580}, R_{680-720}, R_{420-470}, R_{420-550}, R_{420-575}, R_{575-470}, R_{575-550}, R_{540-550}, M, S D, M e \text { and } F\end{array}$ \\
\hline
\end{tabular}

Table 3. Number of Principal Components (PCs) and nearest neighbors (K) used to evaluate k-Nearest Neighbors classification performance using each of the CEFS combinations of spatially-resolved bimodal spectroscopy (each distance D1, D2, D3 and their 4 possible combinations) for spectral features that allowed a pair-wise discrimination of either 6,5 or 4 pairs of histological skin classes

\begin{tabular}{l|ccccccc}
\hline Distance & $\mathrm{D} 1$ & $\mathrm{D} 2$ & $\mathrm{D} 3$ & $\mathrm{D} 1+\mathrm{D} 2+\mathrm{D} 3$ & $\mathrm{D} 1+\mathrm{D} 2$ & $\mathrm{D} 1+\mathrm{D} 3$ & D2+D3 \\
\hline 6 pairs & $\mathrm{PC}=1,2 \ldots, 5$ & $\mathrm{PC}=1,2$ & $\mathrm{PC}=1,2,3,4$ & $\mathrm{PC}=1,2 \ldots, 9$ & $\mathrm{PC}=1,2 \ldots, 6$ & $\mathrm{PC}=1,2 \ldots, 8$ & $\mathrm{PC}=1,2 \ldots, 5$ \\
& $\mathrm{~K}=1,2, \ldots, 15$ & $\mathrm{~K}=1,2, \ldots, 15$ & $\mathrm{~K}=1,2, \ldots, 15$ & $\mathrm{~K}=1,2, \ldots, 15$ & $\mathrm{~K}=1,2, \ldots, 15$ & $\mathrm{~K}=1,2, \ldots, 15$ & $\mathrm{~K}=1,2, \ldots, 15$ \\
$\leq$ 5 pairs & $\mathrm{PC}=1,2 \ldots, 19$ & $\mathrm{PC}=1,2 \ldots, 21$ & $\mathrm{PC}=1,2 \ldots, 30$ & $\mathrm{PC}=1,2 \ldots, 53$ & $\mathrm{PC}=1,2 \ldots, 34$ & $\mathrm{PC}=1,2 \ldots, 42$ & $\mathrm{PC}=1,2 \ldots, 43$ \\
& $\mathrm{~K}=1,2, \ldots, 15$ & $\mathrm{~K}=1,2, \ldots, 15$ & $\mathrm{~K}=1,2, \ldots, 15$ & $\mathrm{~K}=1,2, \ldots, 15$ & $\mathrm{~K}=1,2, \ldots, 15$ & $\mathrm{~K}=1,2, \ldots, 15$ & $\mathrm{~K}=1,2, \ldots, 15$ \\
$\leq 4$ pairs & $\mathrm{PC}=1,2 \ldots, 23$ & $\mathrm{PC}=1,2 \ldots, 33$ & $\mathrm{PC}=1,2 \ldots, 38$ & $\mathrm{PC}=1,2 \ldots, 70$ & $\mathrm{PC}=1,2 \ldots, 47$ & $\mathrm{PC}=1,2 \ldots, 52$ & $\mathrm{PC}=1,2 \ldots, 60$ \\
& $\mathrm{~K}=1,2, \ldots, 15$ & $\mathrm{~K}=1,2, \ldots, 15$ & $\mathrm{~K}=1,2, \ldots, 15$ & $\mathrm{~K}=1,2, \ldots, 15$ & $\mathrm{~K}=1,2, \ldots, 15$ & $\mathrm{~K}=1,2, \ldots, 15$ & $\mathrm{~K}=1,2, \ldots, 15$ \\
\hline
\end{tabular}

Table 4. Number of Principal Components (PCs) used to evaluate Linear Discriminating Analysis (LDA) and Support Vector Machine (SVM) each of the CEFS combinations of spatially-resolved bimodal spectroscopy (each distance D1, D2, D3 and their 4 possible combinations) for spectral features that allowed a pair-wise discrimination of either 6,5 or 4 pairs of histological skin classes

\begin{tabular}{l|ccccccc}
\hline Distance & $\mathbf{D} 1$ & $\mathbf{D} 2$ & $\mathbf{D} 3$ & $\mathbf{D} 1+\mathrm{D} 2+\mathrm{D} 3$ & $\mathrm{D} 1+\mathrm{D} 2$ & $\mathrm{D} 1+\mathrm{D} 3$ & D2+D3 \\
\hline 6 pairs & $\mathrm{PC}=1,2 \ldots, 5$ & $\mathrm{PC}=1,2$ & $\mathrm{PC}=1,2,3,4$ & $\mathrm{PC}=1,2 \ldots, 9$ & $\mathrm{PC}=1,2 \ldots, 6$ & $\mathrm{PC}=1,2 \ldots, 8$ & $\mathrm{PC}=1,2 \ldots, 5$ \\
$\leq$ 5 pairs & $\mathrm{PC}=1,2 \ldots, 19$ & $\mathrm{PC}=1,2 \ldots, 21$ & $\mathrm{PC}=1,2 \ldots, 30$ & $\mathrm{PC}=1,2 \ldots, 53$ & $\mathrm{PC}=1,2 \ldots, 34$ & $\mathrm{PC}=1,2 \ldots, 42$ & $\mathrm{PC}=1,2 \ldots, 43$ \\
$\leq$ 4 pairs & $\mathrm{PC}=1,2 \ldots, 23$ & $\mathrm{PC}=1,2 \ldots, 33$ & $\mathrm{PC}=1,2 \ldots, 38$ & $\mathrm{PC}=1,2 \ldots, 70$ & $\mathrm{PC}=1,2 \ldots, 47$ & $\mathrm{PC}=1,2 \ldots, 52$ & $\mathrm{PC}=1,2 \ldots, 60$ \\
\hline
\end{tabular}




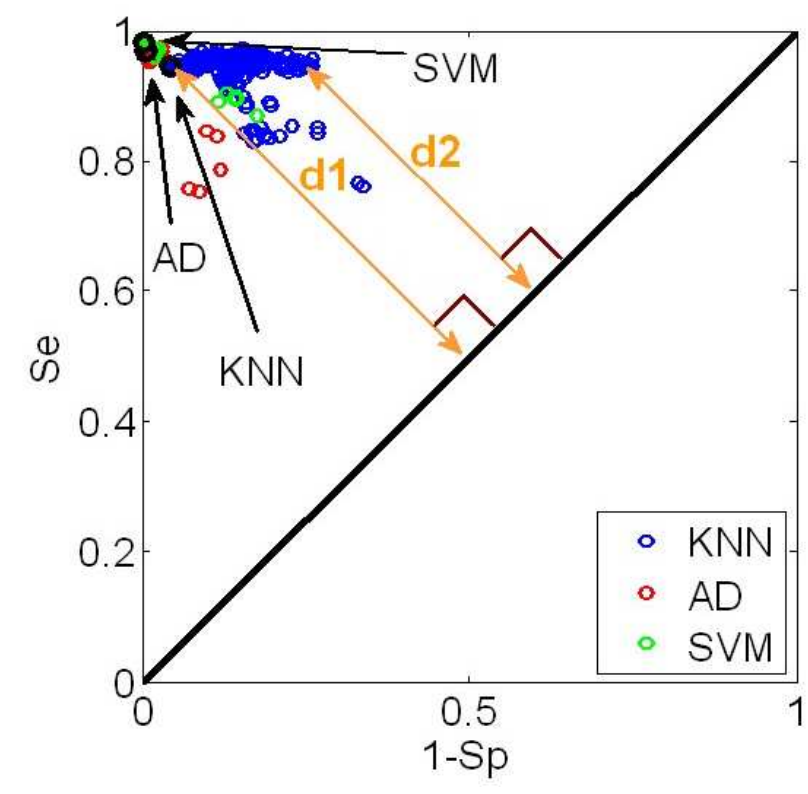

Fig. 3. Receiver Operating Characteristic (ROC) curve. The best global diagnostic performance for the (SE,SP) couple is achieved when the data point is the furthest away from the median line.

ponents (PCs accounting for up to $99 \%$ of the information contained in the original set of data) in order to get the optimum results for sensitivity and specificity. Combinations of the different numbers of $\mathrm{k}$ and PCs retained for each CEFS combinations tested are shown in Table 3. As shown in Table 4, PCs accounting for up to $99 \%$ were taken into account for both LDA and SVM algorithms.

The best classification accuracy achieved by each of the 378 algorithms tested (21 groups of spectral characteristics $\times 3$ methods of classification $\times 6$ pairs of histological classes) i.e. the optimum ( $S e, S p)$ couple is found using a Receiver Operating Characteristic (ROC) test (see Fig. 3) that plots sensitivity as a function of (1 - specificity).

\section{Results}

An example of mean AF spectra for a $410 \mathrm{~nm}$-excitation at the shortest CEFS (D1 $=271 \mu \mathrm{m})$ is given in Figure $4 \mathrm{a})$ for each histological class: $\mathrm{H}(\mathrm{n}=84), \mathrm{CH}(\mathrm{n}=47), \mathrm{AH}(\mathrm{n}=64)$ and $\mathrm{D}(\mathrm{n}=56)$. Significant global intensity differences can be noticed between $\mathrm{H}, \mathrm{CH}$ and $\mathrm{D}$ on one hand and $\mathrm{CH}$ on the other hand. Global intensity is the highest for $\mathrm{H}$, a little lower for $\mathrm{AH}$ and $\mathrm{D}$ (that are close to one another) and the lowest for $\mathrm{CH}$. Focusing on the $500 \mathrm{~nm}$ main emission peak, AH and D display very close intensities (flavins characteristic emission). Typical emission porphyrins double peaks can be observed at 633 and 670 nm. A slight difference between $\mathrm{D}$ and $\mathrm{AH}$ is observed at the 633-nm emission peak (increased intensity for D). An example of mean DR spectra obtained at the shortest CEFS (D1) is given for each histological class $(\mathrm{H}, \mathrm{CH}$, $\mathrm{AH}$ and D) in Figure 4b). Same type of observation as for AF can be made: $\mathrm{CH}$ displays a significantly decreased intensity level than $\mathrm{H}, \mathrm{AH}$ and $\mathrm{D}$. $\mathrm{H}$ displays a significantly decreased intensity compared to both AH and D in the 450-700 nm spectral range, especially for wavelengths longer than $600 \mathrm{~nm}$. It is to be noticed that the global spectral shape of $\mathrm{H}$ classified tissues is very different from the three others. Conversely, intensity and shape of $\mathrm{AH}$ and D mean spectra are very similar. Finally, haemoglobin absorption $577 \mathrm{~nm}$-peak is deeper for $\mathrm{CH}, \mathrm{AH}$ and $\mathrm{D}$ than for $\mathrm{H}$.

Sensitivity $(S E)$ and specificity $(S P)$ of pair-wise classification are given in the left and the right columns respectively of Figures 5 and 6: a) $\mathrm{H}$ vs. $\mathrm{CH}, \mathrm{b}) \mathrm{H}$ vs. $\mathrm{AH}, \mathrm{c}$ ) $\mathrm{H}$ vs. D, d) $\mathrm{CH}$ vs. $\mathrm{AH}, \mathrm{e}) \mathrm{CH}$ vs. D, f) $\mathrm{AH}$ vs. D. Each histogramm displays results for the three classification algorithms (k-NN, LDA and SVM) at each of the possible combinations of CEFS tested: D1 alone, D2 alone, D3 alone, and D1D2D3, D1D2, D1D3, D2D3.

Results in Figure 5 were obtained from a set of 13 characteristics that are common to the discrimination of the 6 pairs of histological classes. Results in Figure 6 were obtained using a set of 186 characteristics that are common to the discrimination of at least 5 pairs of histological classes out of the 6 tested.

No increase in discrimination accuracy was obtained using a more extended set of spectral characteristics (306) common to the discrimination of 4 out of 6 pairs of histological classes compared to the 186 characteristics previously tested. Thus such results are not showed in the current paper.

Using any of the classification algorithm for D1 and for the D1D2D3 combination, very high sensitivy and specificity levels are obtained (close to 100\%) using 13 (D1=6, D2 $=2$ and D3=5) spectral characteristics (Figure 5). When discriminating $\mathrm{H}$ vs. AH and D, both sensitivity and specificity increase ( $S e<90 \%$ and $S p$ close to $100 \%$ ) when using the D1D2D3 combination. In the latter case, sensitivity is higher using the SVM algorithm than the two others. However, both sensitivity (around 65\%) and specificity $(<80 \%)$ are very low when discriminating $\mathrm{AH}$ from $\mathrm{D}$. In that case, it is to be noticed that sensitivity is higher when using the k-NN method than when using the two others; however specificity is higher using D1 and on a global scale, SVM gives the highest results using any of the CEFS combinations.

When considering the 6 pairs of histological classes to discriminate, the D1D2D3 combination reaches higher scores except for the discrimination of AH and D. SVM tends to discriminate healthy $(\mathrm{H})$ from any class of pathologic tissue $(\mathrm{CH}, \mathrm{AH}$ and $\mathrm{D})$ slightly better than k-NN and LDA algorithms. k-NN is slightly more efficient for longest CEFS in discriminating pathologic tissues from one another than LDA and SVM. Such results are always obtained for a large number of PCs, i.e. accounting for more than $99 \%$ of the information contained in the original data set.

Results using the 186 (D1=44, D2=59 and D3=83) characteristics discriminating at least 5 out the 6 pairs of histological classes are shown in Figure 6. Same types of results as the ones using $13(\mathrm{D} 1=6, \mathrm{D} 2=2$ and D3=5) char- 

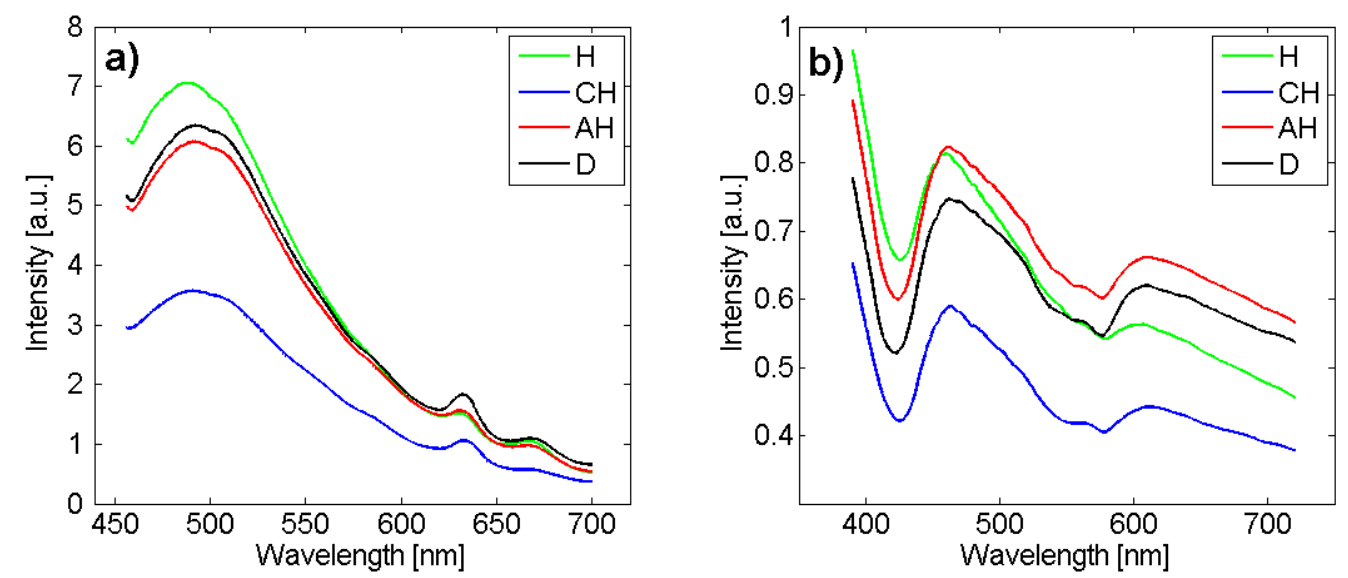

Fig. 4. Mean preprocessed spectra acquired with the developed spectroscopy set-up at the shortest CEFS (D1 $=271 \mu \mathrm{m})$ for each of the four histological classes (H: $n=84, C H: n=47, A H: n=64$ and $D: n=57)$. a) AF spectra obtained for a 410-nm excitation. $b$ ) DR spectra.

acteristics are obtained when discriminating $\mathrm{CH}$ from any other histological class: both sensitivity and specificity get very high (close to $100 \%$ ) using the D1D2D3 combination and any classification algorithm. Results obtained whit extended sets of characteristics (Figure 6) are better than the ones with reduced sets (Figure 5), when considering the use of D2 or D3 alone and when considering the discrimination between $\mathrm{H}$ from $\mathrm{AH}$ and $\mathrm{D}(\mathrm{Se}>95 \%$ and $\mathrm{Sp}$ close to $100 \%$ ) using the D1D2D3 combination. In such cases, LDA and SVM give better results than the k-NN algorithm. Compared to results obtained in Figure 5, a global increase in the discrimination accuracy is noticed when discriminating AH from D: sensitivity is the highest when using D1 alone, D2 alone or D3 alone and specificity is the highest when using any of the three combinations of CEFS. The highest sensitivity $(65 \%)$ is obtained using D1D2D3 with the LDA algorithm. The highest specificity $(80 \%)$ is obtained using the D2D3 combination using the k-NN method (better than both DA and SVM for any CEFS in this case). Neither sensitivity nor specificity is significantly increased compared to the scores obtained an plotted in Figure 5 but they are more "balanced" at each CEFS alone or in combination. Thus the overall accuracy seems better even though it remains low.

The overall classification accuracy is increased especially when discriminating $\mathrm{H}$ vs. $\mathrm{AH}$ and $\mathrm{D}$, using the D1D2D3 combination and using an extended set of spectral characteristics (from 13 to 186). Such best results are obtained for 10 to 20 PCs i.e. accounting for 90 to $95 \%$ of the information contained in the initial set of data.

Finally, sensitivity and specificity are close to $100 \%$ for all pairs of histological classes (except for AH vs. D) using the D1D2D3 combination and for spectral characteristics discriminating at least 5 out of the 6 pairs of histological classes. SVM and LDA seem globally a little more performant than k-NN. However, when discriminating AH from $D$, both sensitivity and specificity remain lower than 70 and $80 \%$ respectively.

\section{Discussion}

Our results confirm and extend other works published namely by Diagardjane et al. [19] in AF multi-excitation on chemically-induced mice skin cancers, by chang et al. [6] and Georgakoudi et al. [29] in bimodal and trimodal spectroscopies (AF multi-excitation and DR) on the cervix or by Inaguama et al. [30] in AF mono-excitation at $410 \mathrm{~nm}$ on human oral cancer. In these studies the authors mentioned that haemoglobin absorption peaks $\approx \approx 420,545$ and $575 \mathrm{~nm}$ ) related to progressive hyperplastic vascular activity and autofluorescence emissions related to flavins, collagen, NADH and porphyrins $(\approx 633$ and $672 \mathrm{~nm})$ can be used as biomarkers to differentiate between various precancerous tissue states. More specifically, the presence of increased porphyrin intensity peaks is also associated with hyperplastic modifications implying haemoglobin transformations [31-33], or changes in the cellular environment [20]. Inaguama et al. [30] also stated that porphyrin may be continuously excreted by cancerous tissue as metabolites, therefore being found not only in advanced cancer but also in early cancer stages.

The spectral characteristics (slopes, ratios, areas, etc.) extracted and selected from our DR spectra are namely related to haemoglobin absorption peaks and those from AF spectra are related to endogenous fluorophores emission spectra of flavins, collagen, NADH and porphyrins. Looking at the averaged and standardized AF emission spectra for excitations at $390,400,410,420$ and $430 \mathrm{~nm}$ we observed that the overall intensity of the mean spectral curve in the emission band from 450 to $550 \mathrm{~nm}$ (related to collagen, NADH and flavins), for healthy classified spectra is higher than the one of the three other classes, for $\mathrm{CH}$ class is lower than the one of the other three classes, for $\mathrm{D}$ is slightly higher than $\mathrm{AH}$ ones. In the wavelength band 550 to $700 \mathrm{~nm}$ (related to flavins and porphyrins), the overall intensity of the mean spectral curve for $\mathrm{CH}$ is higher than the one of the three other classes, for $\mathrm{H}$ 

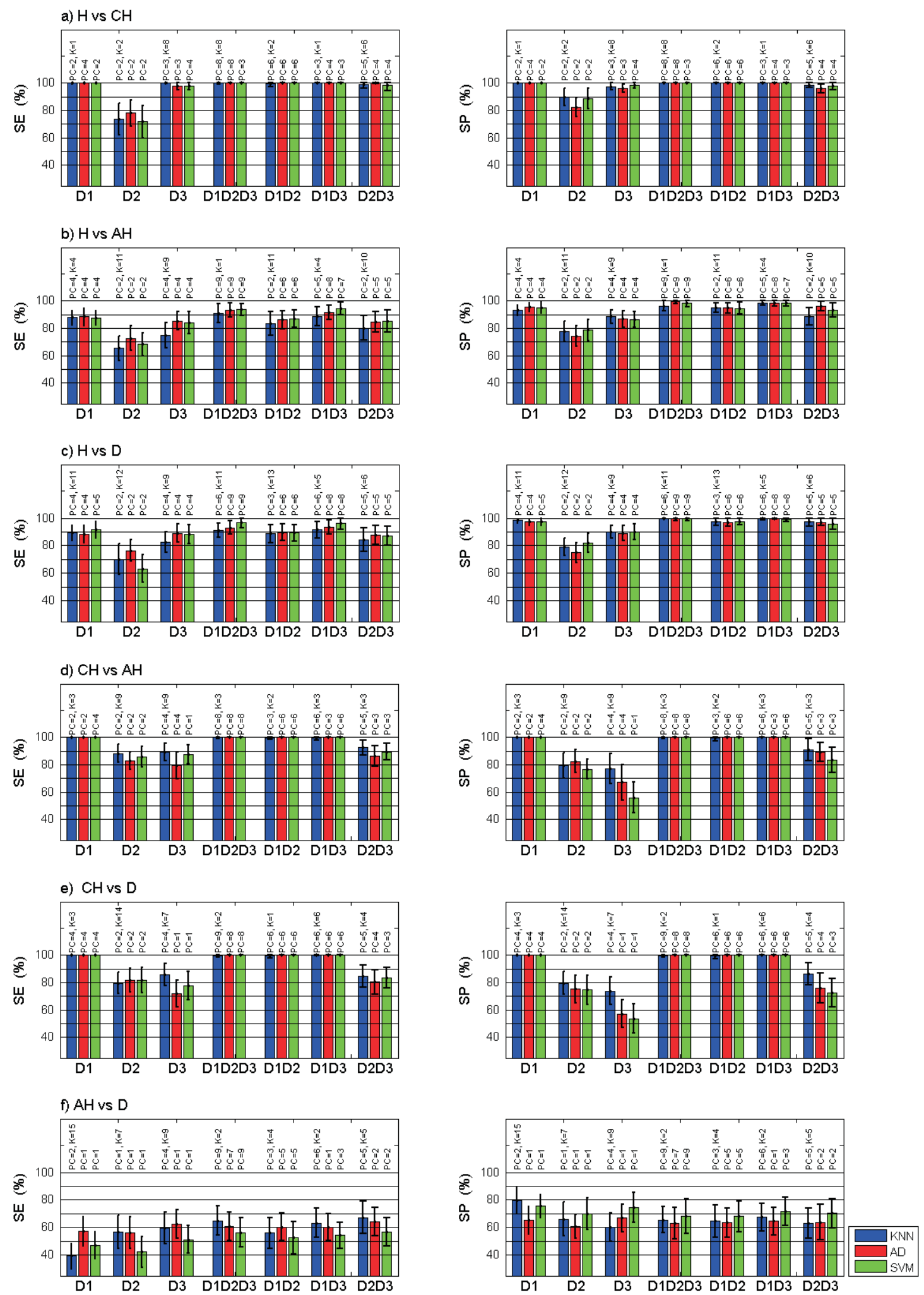

Fig. 5. Mean sensitivity (SE) and specificity (SP) using $13(\mathrm{D} 1=6, \mathrm{D} 2=2$ and $\mathrm{D} 3=5)$ characteristics common to the discrimination of the 6 pairs of histological classes: a) $\mathrm{H}$ (healthy) vs. $\mathrm{CH}$ (Compensatory Hyperplasia), b) H vs. AH (Atypical Hyperplasia), c) H vs. $\mathrm{D}$ (Dysplasia), d) $\mathrm{CH}$ vs. AH, e) $\mathrm{CH}$ vs. D et $\mathrm{f}$ ) $\mathrm{AH}$ vs. D using several combinations of Collection to Excitation Fiber Separation (CEFS): D1 $=271 \mu \mathrm{m}$ alone, D2 $=536 \mu \mathrm{m}$ alone or D3 $=834 \mu \mathrm{m}$ alone, or D1D2D3 or D1D2 or D1D3 or D2D3 and using each of the three classification algorithms: k-NN (k-Nearest Neighbor), LDA (Linear Discriminant Analysis) et SVM (Support Vector Machine). Error bars: standard deviation. PC and K indicated on top of each histogramm correspond to the number of Principal Components (PC) and of neighbors (K) used to obtain optimum results displayed here. 

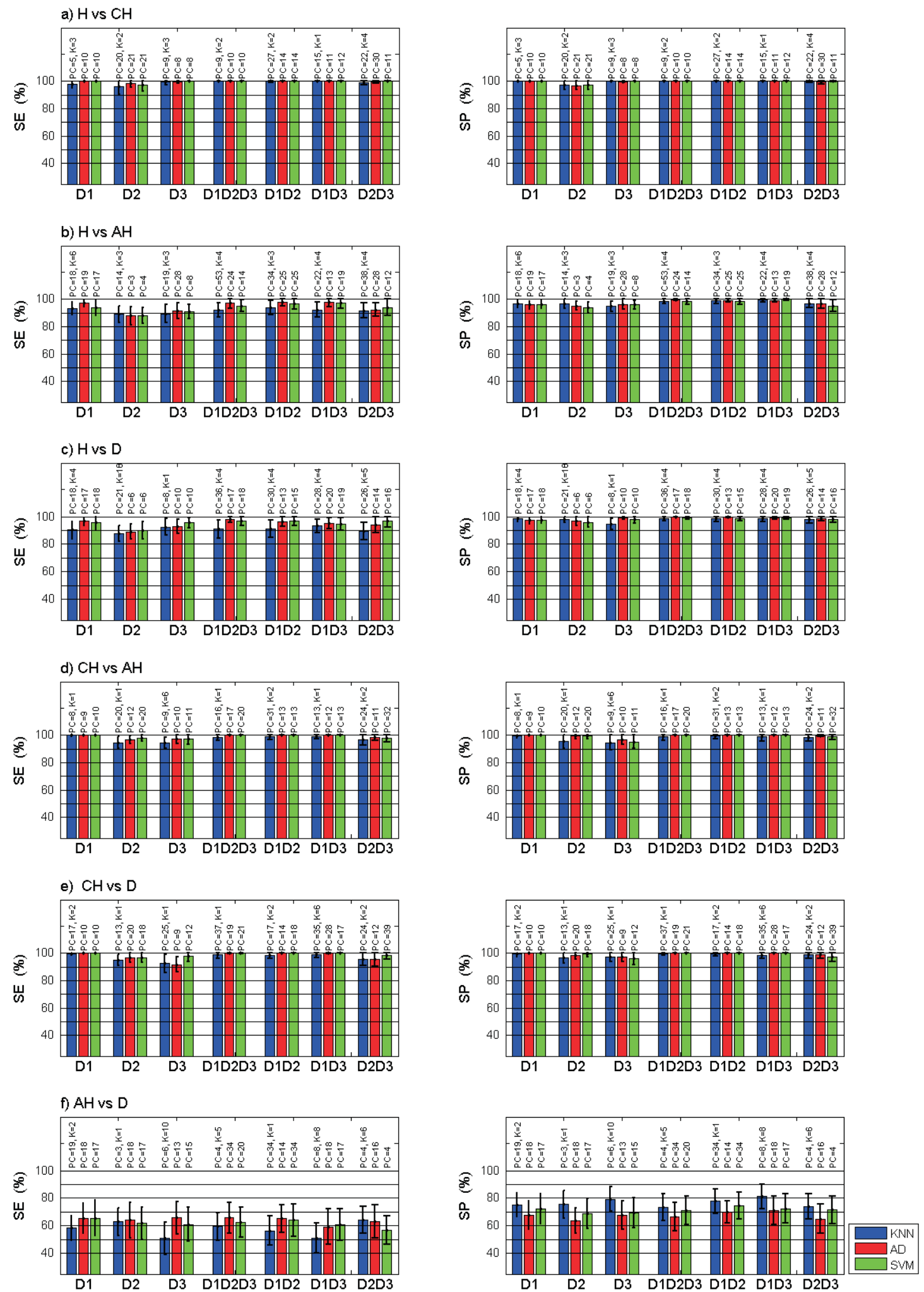

Fig. 6. Mean sensitivity (SE) and specificity (SP) using $186(\mathrm{D} 1=44, \mathrm{D} 2=59$ and $\mathrm{D} 3=83$ ) spectral characteristics common to the discrimination of at least 5 out the 6 pairs of histological classes : a) $\mathrm{H}$ (healthy) vs. $\mathrm{CH}$ (Compensatory Hyperplasia), b) $\mathrm{H} v$ s. AH (Atypical Hyperplasia), c) H vs. D (Dysplasia), d) $\mathrm{CH}$ vs. AH, e) $\mathrm{CH}$ vs. D et f) AH vs. D using several combinations of Collection to Excitation Fiber Separation (CEFS): D1 $=271 \mu \mathrm{m}$ alone, D2 $=536 \mu \mathrm{m}$ alone or D3 $=834 \mu \mathrm{m}$ alone, or D1D2D3 or D1D2 or D1D3 or D2D3 and using each of the three classification algorithms: k-NN (k-Nearest Neighbor), LDA (Linear Discriminant Analysis) et SVM (Support Vector Machine). Error bars: standard deviation. PC and K indicated on top of each histogramm correspond to the number of Principal Components (PC) and of neighbors (K) used to obtain optimum results displayed here. 
class is lower than the one of the three other classes, for $\mathrm{AH}$ class is slightly higher than D ones. For DR spectra acquired at distances D1, D2 and D3, the overall intensity of the mean spectral curve for $\mathrm{CH}$ classified spectra is lower than $\mathrm{AH}$ and $\mathrm{D}$ classes, for $\mathrm{AH}$ class is higher than $\mathrm{CH}$ and D classes, for D class is slightly lower than AH class. The overall intensity of the spectral curve of the class $\mathrm{H}$ is very different from the three others: for D1 in the wavelength band 390-450 nm (haemoglobin absorption peak at $420 \mathrm{Nm}$ ) it is higher than the 3 others; in the band $450-575 \mathrm{~nm}$ it is lower than $\mathrm{AH}$ and in the band 575-720 it is lower than AH and D. Towards D2 and D3 the overall intensity of the mean spectral curve decreases for $\mathrm{H}$ classified spectra: in wavelength band 390-450 it is lower than $\mathrm{AH}$ but of the same amplitude as D; in the band $450-720 \mathrm{~nm}$ it is lower than $\mathrm{AH}$ and D.

While two valleys related to haemoglobin absorption peaks (545 and $575 \mathrm{~nm}$ ) are slightly visible for AF spectra acquired at distance D1, these valleys are more clearly visible (increased) for AF spectra acquired at distance D3; furthermore the emission peak intensity at 633 $\mathrm{nm}$ (porphyrin) is also increased for AF spectra acquired at distance D3. In DR spectra acquired at distance D3, the haemoglobin absorption peaks are increased compared to those acquired at D1. When the distance between excitation-reception fibers increases, the depth of skin probed increases [14], therefore absorption (in DR and AF) and porphyrin emission (in AF) increases (are more visible) for D3.

In a study characterizing early neoplastic changes in DMBA/TPA-induced mouse skin tumor model, using AF multi-excitation in the wavelength band 280-460 $\mathrm{nm}$ and LDA classification, Diagardjane et al. [19] obtained good results in discriminating different precancerous states. They defined 5 categories: category I (Healthy), category II (Inflammation and Hyperplasia), category III (Hyperplasia and Dysplasia), category IV (Moderately Differentiated Squamous Cell Carcinoma) and category V (Poorly Differentiated Squamous Cell Carcinoma). Considering classifications for categories I, II and III using excitations in the wavelength band 360-420, their results were $62.1 \%<S e<69.0 \%$ and $82.2 \%<S p<95.6 \%$. In our work we concentrated on a refined classification within corresponding categories II and III and obtained higher Se and $S p$, while only using NUV-UV excitations wavelengths higher than $360 \mathrm{~nm}$ (i.e. wavelengths with lowest or no mutagenic potential, in perspective of clinical implementation). Chang et al. [6] discriminated healthy (Squamous Normal, SN) vs. Low and High Grades of Squamous Intraepithelial cervical Lesions (LGSIL, HGSIL), using AF multi-excitation in the range $330-480 \mathrm{~nm}$ and DR spectroscopies, in combination with PCA and Mahalanobis distance algorithm. Several pairs of discrimination were defined (SN vs. CN, SN vs. LGSIL, SN vs. HGSIL, CN vs. LGSIL and CN vs. HGSIL). Considering classification of discrimination pairs with SN and LHSIL using AF multiexcitation and DR, their results were $53 \%<S e<95 \%$ and $69 \%<S p<91 \%$. Although our work concerns another type of epithelium, we proposed here a refined classifica- tion of normal and low grades lesions into 4 subclasses $(\mathrm{H}, \mathrm{CH})$ and $(\mathrm{AH}, \mathrm{D})$. Our results are globally higher with Se and $S p>95 \%$ and a lowest discriminating capability for $\mathrm{AH} v s$. D with $\mathrm{Sp} \approx 74 \%$ and $\mathrm{Se} \approx 63 \%$.

Our results (Se and $S p \approx 100 \%$ for $\mathrm{CH}$ vs. others; $S p \approx$ $100 \%$ and $\mathrm{Se}>95 \%$ for $\mathrm{H}$ vs. AH or D; $\mathrm{Sp} \approx 74 \%$ and $S e \approx 63 \%$ for $\mathrm{AH}$ vs. D are also in good agreement with various works that have used classification techniques like SVM or Artificial Neural Networks (ANN). Lin et al. [26] developed an algorithm based on SVM (linear and non linear), using AF excitations at 405 and $436 \mathrm{~nm}$ for discriminating normal and cancerous nasopharyngeal tissues. They achieved $S e=94 \%$ and $S p=97 \%$ using linear SVM and $S e=95 \%$ and $S p=99 \%$ with non linear SVM. PCA can substantially reduce the complexity of an SVM algorithm without sacrificing the performance of the algorithm. But when they combined PCA and SVM methods, these algorithms produced the same results. Nayak et al. [34] developed algorithms based on PCA and ANN, using pulsed laser-induced autofluorescence at $325 \mathrm{~nm}$ excitation for discriminating normal, premalignant and malignant human oral tissues. They achieved $S e=100 \%$ and $S p=92.9 \%$ using linear PCA and $S e=100 \%$ and $S p=96.5 \%$ with ANN. Majunder et al. [35] developed an algorithm based on SVM, using AF excitation at $337 \mathrm{~nm}$ for discriminating healthy squamous $v$ s. early SCC human oral tissues. They achieved $S e=91 \%$ and $S p=96 \%$.

Finally, in order to improve the discrimination efficiency of $\mathrm{AH}$ ws. $\mathrm{D}$ (lowest discriminating capability with $S p \approx$ $74 \%$ and $S e \approx 63 \%$ ), we carried out classification by adding more characteristics which exclusively discriminate $\mathrm{AH}$ $v s$. D to the set of spectral characteristics selected. This approach did not improve significantly the previous results achieved. New methods of nonlinear spectral characteristics selection and nonlinear classification algorithms are to be tested to further investigate increased performance for $\mathrm{AH}$ vs. D classification.

\section{Conclusion}

A simple and tunable instrumentation was developed to acquire both AF and DR spectra in a bimodal approach at several CEFS and applied to monitor healthy to precancerous evolution in vivo of skin mice due to chronic UV-irradiation. Bimodal spectroscopy's accuracy in discriminating four classes of mouse skin histological stages was evaluated. Various sets of spectral characteristics (extracted from the spectral intensity curves) were selected based on statistical discrimination of the pair-wise classes. After data reduction by PCA results obtained with three classification algorithms were compared. Since both sensitivity and specificity are very high when discriminating healthy from any type of hyperplastic tissue on one hand and $\mathrm{CH}$ from $\mathrm{AH}$ and $\mathrm{D}$ on the other hand, bimodal spectroscopy seems a promising tool to help dermatologists and histo-pathologists to increase diagnostic accuracy of skin lesions. However lowest diagnostic accuracy was 
achieved when discriminating AH from D using any of the three linear classification algorithm we tested. It is to be noticed that for these 5 pairs of histological classes, spatial resolution improves classification accuracy and SVM and LDA give better results than the k-NN algorithm. Further studies need to be done to confirm these results on human patients.

\section{Acknowledgments}

This work was supported by the French association Ligue Contre le Cancer (CD 52, 54), Nancy-University, the Region Lorraine and the Programme Alßan, the European Union Programme of High Level Scholarships for Latin America, scholarship No. E05D057651MX.

\section{References}

1. M. Takata, Int. J. Clin 10 (2005) 391-397.

2. M. Rajeswari, Lab. Invest. 83(9) (2003) 1279-1283.

3. J. Oh, J. Biomed. Opt. 11(3) (2006) 032034.

4. I. Bigio, Phys. Med. Biol 42 (1997) 803.

5. V. Tuchin, Handbook of Optical Biomedical Diagnostics, 2002.

6. S. Chang, J. Biomed. Opt. 10(2) (2005) 024031.

7. D. Veld, Lasers Surg. Med. 36 (2005) 356.

8. I. Georgakoudi, Gastroenterol. 120 (2001) 1620.

9. U. Utzinger, J. Biomed. Opt. 8(1) (2003) 121.

10. C. Zhu, J. Biomed. Opt. 10(02) (2005) 024032.

11. J. Tunnell, Technol. Cancer Res. Treat. 2 (2003) 505.

12. R. Zangaro, Appl. Opt. 35 (1996) 5211.

13. A. Zuluaga, Appl. Spectrosc. 53 (1999) 302.

14. K. Katika, Appl. Opt. 45(17) (2006) 4174-4183.

15. D. Veld, J. Biomed. Opt. 9(5) (2004) 940-950.

16. N. Marín, J. Biomed. Opt. 11(1) (2006) 014010.

17. E. Péry, Ph.D. thesis, Institut National Polytechnique of Lorraine (2007).

18. V. Wallace, Phys. Med. Biol. 45 (2000) 735-751.

19. P. Diagardjane, Lasers Surg. Med. 37 (2005) 382.

20. S. Balasubramanian, Carcinogenesis 16 (1995) 2461.

21. B. Murphy, J. Biomed. Opt. 10(6) (2005) 064020.

22. N. Marín, Gyneco. Oncol. 99 (2005) S116-S120.

23. S. Kamath, J. Biomed. Opt. 12(1) (2007) 014028.

24. D. Peña, Análsis de datos multivariantes, 2002.

25. N. H. Timm, Applied multivariate analysis, 2002.

26. W. Lin, J. Biomed. Opt. 9(1) (2004) 180-186.

27. C. Burges, Data Min. Knowl. Discov. 2 (1998) 121-167.

28. V. Vapnik, The Nature of Statistical Learning Theory, 1995.

29. I. Georgakoudi, Am. J. Obstet. Gynecol. 186(3) (2002) 374382.

30. M. Inaguma, Cancer 86 (1999) 32201-11.

31. Y. Yang, Lasers Surg. Med. 7 (1987) 528-532.

32. Z. Malik, Cell Differentiation 8 (1979) 223-33.

33. T. Nishisaka, J. Jpn. Soc. Laser Med. 16 (1995) 11-15.

34. G. Nayak, Biopolumers 82 (2006) 152-166.

35. S. Majumder, Lasers Surg. Med. 36 (2005) 323-333. 